\title{
III \\ EL NUEVO REGLAMENTO DEL SENADO
}

Referencia de los trabajos de la Cámara del Senado de elaboración del:

Reglamento del Senado: El artículo 72.1 de la Constitución española de 1978 determina que las Cámaras establecen sus propios reglamentos, requiriéndose para su aprobación la mayoría absoluta de la Cámara.

1. INICIATIVA:

PROPOSICION DE LEY DEL REGLAMENTO DEL SENADO

B.O.C.G.

Fecha

13 (a) Con fecha 26 de junio de 1980 , el presidente de la Comisión de Reglamento comunica a la Presidencia del Senado la conclusión de los trabajos de elaboración de un proyecto de Reglamento del Senado por la Ponencia constituida dentro de dicha Comisión e integrada por los señores Ballarín Marcial, Benito Calleja, García Oliva, Lizón Giner, Nieves Borrego, Rodríguez de la Barbolla y Villar Arregui. La Mesa del Senado, reunida el 1 de julio de 1980, dispone la publicación del proyecto en el Boletín Oficial de las Cortes Generales, dando un plazo de diez días para presentación de las enmiendas, que se iniciaría el 1 de septiembre, finali-

13 (b) zando el 11 de septiembre, fecha ésta que, una vez expirada, experimentaría una ampliación de cinco días, haciendo uso del derecho contemplado en el artículo 87.2 del anterior Reglamento provisional del Senado.

\section{ENMIENDAS PRESENTADAS}

13 (c) Las enmiendas presentadas al proyecto, en cumplimiento de lo dispuesto en el artículo 149 del anterior Reglamento, fueron 390 , por parte de los distintos Grupos Parlamentarios: Mixto, U. C. A.; Senadores Vascos, Socialistas, Socialista Andaluz y Catalunya; Democracia i Socialisme.

Al art. 1.: Enmiendas núms. 61, 109, 110, 244.

$\gg$ 2.: Enmienda núm. 111.

$\gg$ \3.: Como art. nuevo, enm. núm. 380.

$\gg \quad 40^{\circ}:$ Enm. núms. 62, 245, 246.

$\gg 770^{\circ}:$ » 63 .

10 sept. 1980

8 julio 1980 
B.O.C.G.

$13(c)$

\begin{tabular}{|c|c|c|c|}
\hline$\gg$ & $\gg$ & 11: & $\gg$ \\
\hline » & $\gg$ & 12: & $\gg$ \\
\hline$\gg$ & $\gg$ & 13: & $\gg$ \\
\hline$\gg$ & $\gg$ & 15: & $\gg$ \\
\hline$\gg$ & $\gg$ & 16: & $\gg$ \\
\hline$\gg$ & $\gg$ & 17: & $\gg$ \\
\hline$\gg$ & $\gg$ & 18: & $\gg$ \\
\hline$\gg$ & $\gg$ & 19: & $\gg$ \\
\hline$\gg$ & $\gg$ & 20: & $\gg$ \\
\hline$\gg$ & $\gg$ & 21: & 》 \\
\hline$\gg$ & $\gg$ & 23: & $\gg$ \\
\hline
\end{tabular}

$64,65,112,156,247$ 379.

》 $66,113,140,248$.

$\gg 67,232,249,340$.

$\gg 1,68$.

$\gg 69$.

36.

$56,70,233,250,251$.

1.

$36,42,114,157,252$.

$1,253,254,341$.

$36,141,142,158,159$, $160,255,256,257$, $258,259,282,385$.

$\gg \quad \gg \quad \gg 1,36,16 \mathrm{i}, 234,260$, 386.

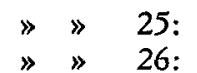

Capítulo II:

$\mathrm{Al}$ art. 27:

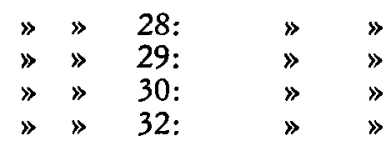

2, 36, 54, 235, 237, 261. $36,262$. 117,342 . $3,4,29,30,36,37,53$, $115,116,162,163$, $164,263,284,383$.

$71,175,265$.

266.

$72,73,236,267,283$. $5,6,7,36,38,52,57$, $58,74,118,166,268$, 344.

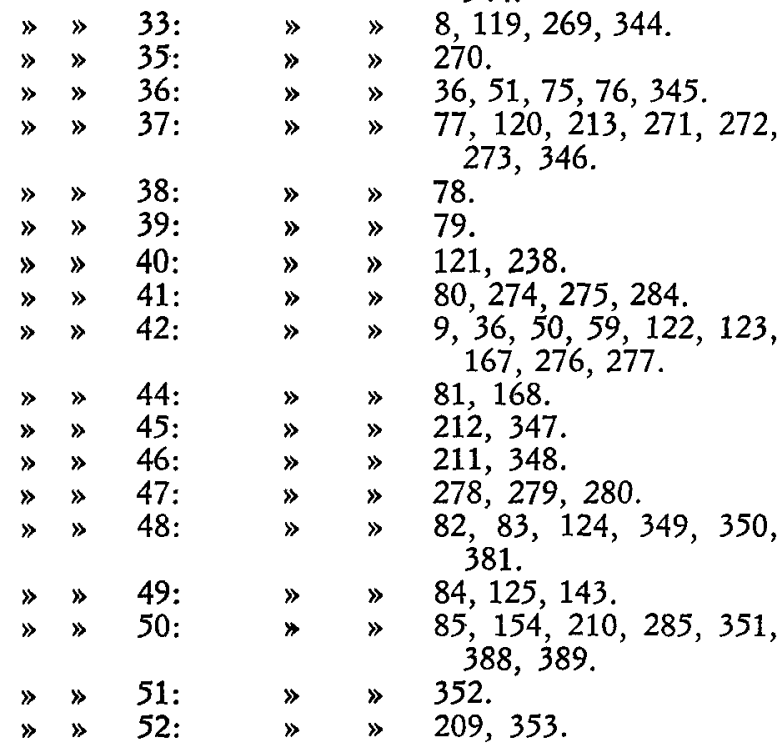


B.O.C.G.

$13(c)$

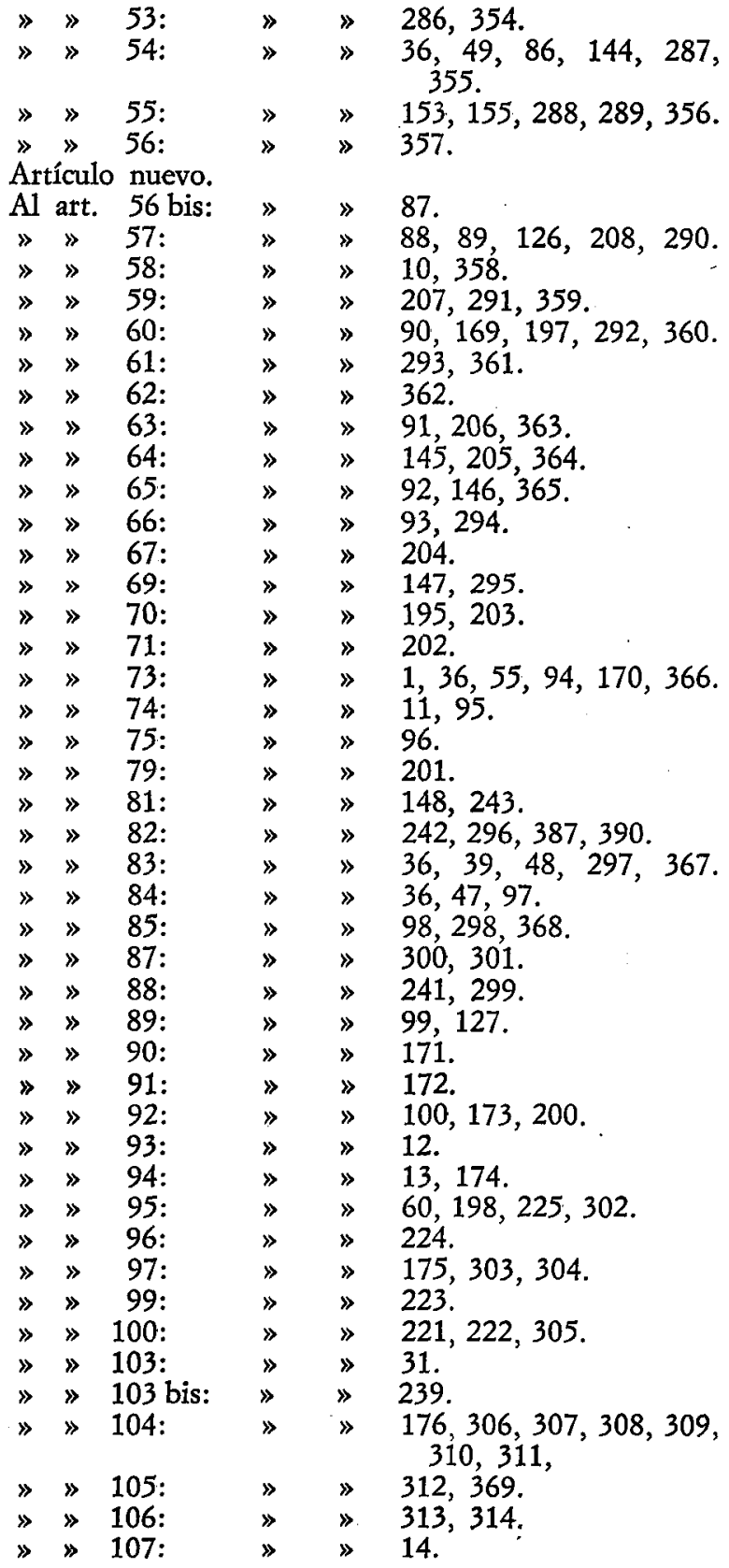


B.O.C.G.

13 (c)

$\gg \gg 108: \quad \gg 15,101,177,315,370$.

$\gg \gg 110$ :

$\gg \gg 111:$

$\gg$ 112:

$\gg$ 113:

$\gg$ 116:

$\gg \gg 117:$

$\gg$ 119:

\ 120:

$\gg$ \120 bis:

$\gg \gg 120$ bis: $\gg$ \$ $\gg 319$.

$\gg 178,193$.

102,220 .

$103,179$.

$128,316$.

$16,180,196,219,371$.

17.

$149,194$.

$181,317,318$.

$\gg$ \122 bis (nuevo): » $18,321$.

$\gg \gg 122$ ter (nuevo): » 19.

$\gg \gg 125: \gg \gg 104$.

$\gg \gg 125$ bis: $\gg \gg 192$.

$\gg \gg 126: \gg \gg 129$.

$\gg \gg 130: \quad \gg 218$.

$\gg$ 131:

$\gg$ 132:

$\gg$ 133:

$\gg 135$ :

$\gg$ 136:

Cap. II, Sec. $5 .^{\mathrm{a}}$ (nuevos)

$\mathrm{Al}$ art. 136 bis: 》

$\gg$ 》 137:

$\gg 139$ :

$\gg 140$ :

$\gg$ 142:

$\gg$ 143:

\ 144:

$\gg 145$ :

$\gg$ 146:

$\gg$ 152:

$\gg \quad \gg 32,150,217,372$.

$32,150,217,372$
$20,36,46,182$

$21,36,45,183$.

$22,184$.

185.

Tít. VI (nuevo): » »

Al art. 153 bis (nuevo): "

» $\gg 154$

$\gg$ 155:

$\gg$ 156:

$\gg$ 157:

$\gg$ 158:

\ 161:

$\gg$ 162:

$\gg$ 163:

\ 164:

Título VII:

$\mathrm{A} 1$ art. 166:

$\gg$ 167:

$\gg$ 168:

$186,322$.

$187,188$.

138.

105.

33,323 .

$216,324,325,326$.

215, 327:

$130,328,373,382$.

214.

$329,330,374$.

131,331 .

189.

189.

151.

$24,332$.

$25,132,230,333,334$.

229.

26.

106, 152, 231.

199.

$27,227,228,375$.

$28,107,335,376$.

108.

336.

337.

$337,381$. 
B.O.C.G.

$13(c)$

\begin{tabular}{|c|c|c|c|c|}
\hline$\gg$ & 169: & $»$ & $"$ & 377. \\
\hline$»$ & 174: & $»$ & $\gg$ & 226,338 . \\
\hline$»$ & $>175:$ & $\gg$ & $»$ & $36,40,44,190$ \\
\hline$\gg$ & > 176: & $\gg$ & $»$ & $1,133,384$. \\
\hline$\gg$ & $>177:$ & $»$ & $»$ & 378. \\
\hline$\gg$ & 178: & $\gg$ & $»$ & $134,339$. \\
\hline$»$ & 179: & $»$ & $»$ & 135. \\
\hline$\gg \otimes$ & 180: & $\gg$ & $»$ & 136. \\
\hline$\gg \gg$ & > 180 bis: & $»$ & $»$ & 137. \\
\hline Disp. & - adicional & primera & & $138,383$. \\
\hline Disp. & - transitoria & primera & & $34,41,139$. \\
\hline$»$ & $\gg$ & segunda & & 36,43 \\
\hline 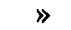 & $»$ & cuar & & \\
\hline
\end{tabular}

B.O.C.G.

Fecha

13 (d) Corrección de erratas de las enmiendas presentadas, a instancias del senador Portabella Rafols, cuya enmienda núm. 53 sufrió un etror.

\section{INFORME DE LA PONENCIA}

13 (e) En cumplimiento de lo dispuesto en el artículo 149 de 1 junio 1981 del Reglamento provisional del Senado, se procedió a la publicación, en el Boletín Oficial de las Cortes Generales, del informe emitido por la ponencia designada en el seno de la Comisión de Reglamento sobre el Proyecto de Reglamento del Senado. Dicha ponencia estuvo integrada por los senadores don Armando Benito Calleja, don Manuel Fombuena Escudero, don Arturo Lizón Giner, don Julio Nieves Borrego, don Antonio Ojeda Escobar, don Joan Prats i Catalá y don Francisco Villodres García, asistida por el letrado don Fernando Santaolella López, aceptando. dicha ponencia un gran número de enmiendas.

\section{DICTAMEN DE LA COMISION}

13 (f) La Comisión de Reglamento, a la vista del informé de 29 abril 1982 la ponencia designada para el estudio del proyecto, emitió su dictamen, publicándose asimismo los votos particulares presentados al dictamen emitido por la Comisión.

Los votos particulares que experimentó fueton 18, por parte de los distintos Grupos Parlamentarios: Catalunya, Democracia i Socialisme (C.D. I. S.), Senadores Vascos (S. V.), Mixto (MX), Socialista (S), Socialista Andaluz (S. A.), Unión de Centro Democrático (UCD). 


\section{DEBATE EN EL PLENO DEL DICTAMEN \\ DE LA COMISION}

D.S.S.

Fecba Págs.

N. 156 Arts. 1.0 a 21: Habiendo recaído los votos particulares, fueron aprobados por asentimiento de la Cámara. señor Unzueta Uzcanga, correspondiente a la enmien-

$26-5-82 \quad 7752$

$26-5-82 \quad 7752$ da núm. 158 , se somete a la votación de la Cámara con dicha modificación, siendo aprobado.

» Arts. 23 a 26: Fueron aprobados por asentimiento de la Cámara.

» Art. 27: Se rechaza el voto particular del señor Bosque Hita, correspondiente a la enmienda núm. 3; retirando a continuación otro voto particular correspondiente a la enmienda núm. 4, fue aprobado el texto del dictamen por votación.

» Arts. 28 a 31: No habiendo sido objeto de votos par-

$26-5-827763$ ticulares, fue aprobado por asentimiento de la Cámara.

Art. 32: El voto particular del señor Bosque Hita,

$26-5-82$

7763 que se correspondió con una enmienda in voce, fue rechazado, sometiéndose a continuación el texto del dictamen a votación, fue aprobado.

» Arts. 33 a 36: Fueron aprobados por asentimiento de la Cámara tras ser retirados los votos particulares formulados.

Arts. 37 a 48: Sometidos directamente a la decisión

26-5-82 7755

$26-5-82 \quad 7755$ de la Cámara, fueron aprobados por asentimiento de la misma.

Art. 49: El voto particular del señor Cercos Pérez, correspondiente a la enmienda núm. 86 , fue aprobado, y sometido a votación el texto del dictamen, con la modificación mencionada, fue aprobado por unanimidad.

Art. 50: Fue aprobado por asentimiento.

26-5-82 7767

Arts. 51 a 64: Retirados los votos particulares formu-

$26-5-827767$ lados, fueron aprobados por asentimiento de la Cámara.

Arts. 65 y 66: Retirados los votos particulares formu-

$26-5-82$

7767 lados, fueron aprobados por asentimiento.

Art. 67: Se aprueba el texto del dictamen, incluyén-

$26-5-827767$ dose una enmienda transaccional.

Arts. 68 a 94: Fueron aprobados directamente.

Arts. 95 a 107: Aprobados conforme al texto del 26-5-82 7768 dictamen.

Art. 108: Rechazado el voto particular del señor Villo-

$26-5-82$

7768

dres García, correspondiente a una enmienda in voce, fue aprobado el texto del dictamen. lares, por lo que fueron aprobados por asentimiento. Arts. 115 a 126: Fueron aprobados. 
D.S.S.

Fecha Págs.

$26-5-82 \quad 7776$

Art. 127: Quedó suprimido este precepto por asentilos portavoces de los Grupos.

$\gg \quad$ Arts. 128 y 129: No siendo objeto de votos particulares, fueron aprobados.

» Art. 130: Tras la propuesta de un texto transaccional por parte de los portavoces de los Grupos Parlamentarios, fue aprobado el nuevo texto. $\gg \quad$ Arts. 131 a 138: Fueron aprobados.

26-5-82 7777

$26-5-82 \quad 7777$

26-5-82 7777

Art. 139: El señor Bosque Hita presentó dos votos particulares que se correspondían con las enmiendas núms. 21 y 22; aprobándose por la Cámara el voto particular de la enmienda núm. 21, quedando así modificado el presente artículo.

Art. 140: Fue aprobado.

26-5-82 7778

Art. 141: El segundo voto particular del señor Bosque Hita, correspondiente a la enmienda núm. 22, fue $26-5-827778$ aprobado por unanimidad, siendo, pues, aprobado el texto del dictamen con la modificación anterior.

" Arts. 142 a 149: Fueron aprobados por asentimiento.

$» \quad$ Art. 150: Fue aprobado el texto del dictamen al ser rechazado el voto particular del señor Subirats Piñona correspondiente a una enmienda in voce.

Art. 151: Fue aprobado sin discusión.

» Art. 152: Los portavoces de los Grupos Parlamentarios presentaron un nuevo texto, que fue aprobado por la Cámara.

$\gg \quad$ Arts. 153 a 175: Fueron aprobados por asentimiento, no habiendo sido objeto de voto particular alguno.

" Art. 176: Fue aprobado con la modificación de la pro. puesta formulada, postulando la adición de un inciso al párrafo primero.

» Arts. 177 a 197: A propuesta del señor presidente, fueron aprobados por asentimiento.

» Disposición transitoria quinta: Se aceptó una propuesta de modificación formulada por los portavoces de los distintos Grupos Parlamentarios.

" Disposición transitoria sexta: No siendo objeto de votos particulares, se procedió a su aprobación.

》 Disposición final: Sufrió una modificación, a propuesta $26-5-82 \quad 7782$ de los portavoces de los Grupos Parlamentarios.

Procediendo a continuación a un análisis comparativo entre el índice sistemático del Proyecto de Reglamento del Senado, presentado por el presidente de la Comisión de Reglamento, y el definitivo texto propuesto para la aprobación de la Cámara, podemos señalar las siguientes observaciones:

- Título primero: De la Constitución del Senado. No sufre esencialmente alteración alguna, exceptuando la formación del capítulo II, que se dirá: Del procedimiento para la Constitución del Senado.

- Título segundo: De los senadores y de los Grupos Parlamentarios. No se 
modificaría más que el capítulo I, que se mencionaría: De las prerrogativas y obligaciones parlamentarias de los senadores.

- Título tercero: De la organización y funcionamiento del Senado. Sólo se modificaría el capítulo IV: De las Comisiones que experimentarían una más amplia división en cinco secciones.

- Título cuarto: Del procedimiento legislativo. El capítulo I no se alteraría, pero el capítulo II: De los procedimientos legislativos especiales, experimentaría las siguientes modificaciones: Se añade una sección primera referente a la tramitación de un proyecto de ley en lectura única.

Esta innovación haría correr así el orden de las posteriores secciones. Añadiéndose igualmente una sección sexta: De los Estatutos de Autonomía; se convierte en Sección séptima lo que inicialmente fueta capítulo III: De los Tratados y Convenios internacionales.

El antiguo capítulo IV: Del procedimiento presupuestario, pasaría así a ser capítulo III.

- Título quinto: Del procedimiento de revisión constitucional. Quedaría sustancialmente modificado, al incluir dentro de este título un capítulo I: Sobre la revisión constitucional iniciada en el Senado. Un capítulo II: Sobre la revisión Constitucional iniciada en el Congreso. Un capítulo III: Sobre la reforma constitucional prevista en el artículo 168 de la Constitución.

- Título sexto: De los ruegos, preguntas e interpelaciones. Desaparecería en el texto definitivo la palabra «ruegos». En lo que respecta a su capítulo I, se añadirían tres secciones: sección primera: Normas generales; sección segunda: De las preguntas de contestación oral, y sección tercera: De las preguntas de contestación escrita.

- Título séptimo: De las mociones. Quedaría igual.

- Título octavo: Pasaría al título noveno, siendo el definitivo título octavo: De las Comunicaciones e informes del Gobierno y de otros órganos estatales.

- Título noveno.: El anterior título noveno pasaría al título décimo, quedando, pues, el título noveno como sigue: De las relaciones del Senado con otras instituciones constitucionales. Constando el presente título de cuatro capítulos y no de tres, siendo el nuevo capítulo añadido el III, que hace referencia a la elección de otros órganos.

- Título décimo: Sufre asimismo modificación, quedando: De la publicidad de los trabajos del Senado.

- Título undécimo: Sigue experimentando la ulterior modificación numérica, quedando, pues, el presente título como: De las peticiones.

- Título duodécimo: De la reforma del Reglamento. El presente título duodécimo (en el texto inicial eran 11) aparece como consecuencia del corrimiento numérico que se viene arrastrando.

Finalmente, en el texto definitivo se contemplará una disposición final, no prevista en el texto del Proyecto.

Finalizado el debate del articulado y, en cumplimiento de lo dispuesto en el artículo 72.1 de la Constitución, el señor presidente propuso que la Cámara se pronunciara sobre la totalidad del dictamen del nuevo Reglamento del Senado, que para ser aprobado requeriría la mayoría absoluta de sus miembros.

Finalizada la votación, fue aprobado definitivamente el nuevo Reglamento de la Cámara con el voto favorable de 138 senadores, en fecha 26 de mayo de 1982; ordenándose su publicación en el Boletín Oficial de las Cortes Generales, número $13(\mathrm{~g})$, de 4 de junio de 1982 .

M.a Victoria García-Atance 